\title{
EDICIÓN ESPECIAL
}




\section{revie}

Revista Digital de suscripción gratuita del Instituto Dominicano de Evaluación e Investigación de la Calidad Educativa (IDEICE)

Periodicidad Semestral

Edición

Febrero 2019, Vol. 6, No. 1

Dirección Ejecutiva

Julio Leonardo Valeirón Ureña

Consejo Editorial

Dinorah de Lima Jiménez

Julián Álvarez Acosta

Luis Camilo Matos De León

Dilcia Armesto Nuñez

Luis Emilio Segura

Liliana González

Evaluadores

Pascuala Matos

Rosa Oviedo

Cheila Valera

Santa Cabrera

Julián Álvarez

Correción de estilos

Luis Emilio Segura

Coordinación

Liliana González

Dirección

Dilcia Armesto Núñez

Colaboración

Lidia Moreta

Francisco Martínez Cruz

Diseño y Diagramación

Natasha Mercedes Arias

Yeimy R. Olivier Salcedo

ISSN: 2409-1553 (Digital)

2636-2260 (Impreso)

IDEICE

Calle José Andrés Aybar Castellanos No.79

(Prolongación México),

La Esperilla, Santo Domingo, D,N.

Teléfono: +1 (809) 732-7152

www.ideice.gob.do
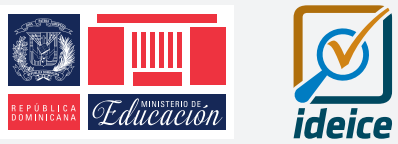

Esta obra está bajo una licencia de Creative Commons Atribución-NoComercial-SinDerivar 4.0 Internacional

\section{PRESENTACIÓN}

El Instituto Dominicano de Evaluación e investigación de la Calidad Educativa (IDEICE), en alianza con el Programa de Capacidades LAC Reads (PCLR) de la Agencia de los Estados Unidos para el Desarrollo Internacional (USAID), presenta a la comunidad educativa el número especial de su Revista Revie, donde se exponen los resultados de investigaciones en lectoescritura para el nivel inicial en República Dominicana y otros países de la región de América Latina y el Caribe. El PCLR tiene como objetivo aumentar el impacto, la escala y la sostenibilidad de las intervenciones de lectoescritura inicial (LEI) a través de la generación y diseminación de evidencia y formación de capacidades locales.

En primera instancia se presenta "RedLEI: uniendo voluntades para desarrollar investigación sobre lectoescritura inicial en contexto", un artículo de tipo narrativo que describe cómo el PCLR ha creado una red de universidades en Centroamérica y República Dominicana, y cómo integra esfuerzos inéditos con el organismo regional (CECC-SICA) para investigar y crear capacidades locales en investigación en LEl para generar conocimiento e informar la toma de decisiones de políticas públicas educativas.

La situación actual en el área de Lectoescritura de educación inicial tanto en nuestro país como en Latino- Centroamérica y el Caribe se refleja en los artículos subsiguientes "Lectoescritura inicial en Latinoamérica y el Caribe: una revisión sistemática" que detalla más a fondo la metodología que utilizó el PCLR para la revisión sistemática sobre el LEl, realizada en fecha del 1995 hasta el 2016, donde se aprecia cuál es la realidad lectora de niños de esta región. "Lectoescritura inicial en Centroamérica y República Dominicana: aprendizajes, recomendaciones y desafíos", este artículo, ofrece propuestas para la construcción de una agenda de investigación en lectoescritura del nivel inicial e identifica áreas potenciales de trabajo para los actores clave que intervienen en ese campo.

Continúa la revista con dos investigaciones que utilizan la técnica de mapeo con los títulos "Mapeo y análisis de actores clave en lectoescritura inicial del Programa de Capacidades LAC Reads (2016-2018)", artículo que despeja algunas interrogantes, para determinar en que debe basarse la investigación sobre el LEl y así definir los intereses de cada país del área para mejorar sus capacidades lectoras.

El subsiguiente artículo "Actores clave en lectoescritura inicial en República Dominicana: resultados de un mapeo y análisis de actores" describe las opiniones de los actores que fueron entrevistados durante los años 2016-2017 y que permitieron señalar las fortalezas y desafíos como país e identificar las necesidades de formación de capacidades en el aprendizaje de LEI.

Por último, se presenta además la utilización de los datos "PISA 2015 en República Dominicana", para medir la relación entre los años de educación inicial y los logros educativos y el desempeño en PISA, así como algunas recomendaciones de estudios y políticas educativas a seguir.

Julio Leonardo Valeirón Ureña

Director Ejecutivo 


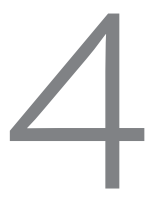

REDLEI: UNIENDO VOLUNTADES PARA DESARROLLAR INVESTIGACIÓN SOBRE LECTOESCRITURA INICIAL EN CONTEXTO

Ana Patricia Elvir

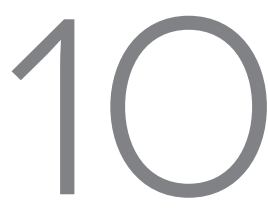

LECTOESCRITURA INICIAL EN CENTROAMÉRICA

Y REPÚBLICA DOMINICANA: APRENDIZAJES, RECOMENDACIONES Y DESAFÍOS

Josefina Vijil

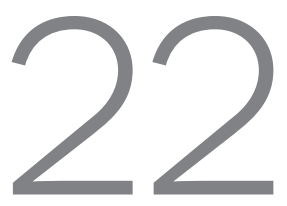

LECTOESCRITURA INICIAL EN LATINOAMÉRICA Y EL CARIBE: UNA REVISIÓN SISTEMÁTICA

Rebecca Stone

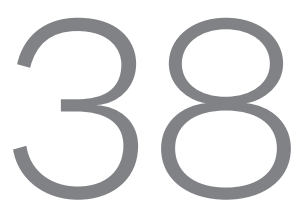

ACTORES CLAVE EN LECTOESCRITURA INICIAL EN REPÚBLICA DOMINICANA. RESULTADOS DE UN MAPEO Y ANÁLISIS DE ACTORES

Josefina Vijil

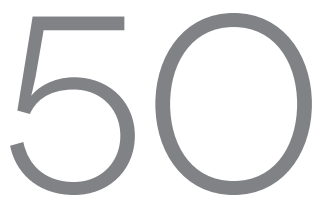

MAPEO Y ANÁLISIS DE ACTORES CLAVE EN LECTOESCRITURA INICIAL DEL PROGRAMA DE CAPACIDADES LAC READS DE USAID (2016-2018)

Yesly Contreras

UNA APROXIMACIÓN A LOS RETORNOS EN LOGROS

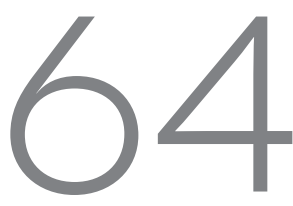
EDUCATIVOS DE LOS AÑOS DE EDUCACIÓN INICIAL USANDO LOS DATOS DE PISA 2015 PARA REPÚBLICA DOMINICANA 



\section{LECTOESCRITURA INICIAL EN CENTROAMÉRICA Y REPÚBLICA DOMINICANA: APRENDIZAJES, RECOMENDACIONES Y DESAFÍOS}

\section{RESUMEN}

El presente artículo pretende detallar brevemente lo -que hemos aprendido acerca de la Lectoescritura Inicial en Centroamérica y República Dominicana, sus potencialidades y desafíos, a través de los diferentes componentes del Programa de Capacidades LAC Reads (PCLR) a lo largo de 3 años de ejecución. A partir de lo estudiado, ofrece propuestas para la construcción de una agenda de investigación en lectoescritura inicial en la región e identifica áreas potenciales donde el programa podría expandirse y desarrollar su trabajo.

\section{PALABRAS CLAVE}

Lectoescritura inicial en Centroamérica y República Dominicana, evidencias, desarrollo de capacidades.

\section{ABSTRACT}

This article aims to briefly detail what we have learned about early grade literacy in Central America and the Dominican Republic, its potential and challenges, through the different components of the LAC Reads Capacity Program (LRCP) during its 3 years of implementation. Based on what has been studied, it offers proposals for the construction of an early grade literacy research agenda in the region and identifies potential areas where the program could expand and develop its work.

\section{KEYWORDS}

Early grade literacy in central america and the Dominican Republic, evidence, capacity-building. 


\section{INTRODUCCIÓN}

Centroamérica ha sido siempre una región con grandes potencialidades, una tierra de lectores y escritores insignes, Rubén Darío es un ejemplo de ello. De esta región provienen autores que han ganado los premios más importantes en lengua castellana: Miguel Ángel Asturias, Premio Nobel de Literatura, Ernesto Cardenal y Claribel Alegría, premio "Reina Sofía de Poesía Iberoamericana" y Sergio Ramírez, Premio de Literatura en Lengua Castellana "Miguel de Cervantes". Sin embargo, esa potencialidad no se ha recogido en la enseñanza en las escuelas. Aunque la región ha producido grandes poetas y narradores, la calidad de la educación de nuestros países es deficitaria, y nuestros niños y niñas no están aprendiendo a leer y escribir bien desde los primeros grados. En las escuelas no estamos aprovechando, ni desarrollando ese potencial. Hacer frente a esta realidad es un reto en la región.

De eso se trata el trabajo que realizamos mediante este Programa.

\section{EL PROGRAMA DE CAPACIDADES LAC READS}

El Programa de Capacidades LAC Reads (PCLR), de la Oficina de USAID para América Latina y el Caribe, es ejecutado con el apoyo del American Institutes for Research (AIR), en asociación con Juárez y Asociados (J\&A) y la participación de organizaciones socias a nivel nacional. Se ha estado ejecutando desde 2014 y durará hasta el 2019, priorizando Guatemala, Honduras, Nicaragua, Perú, Haití, República Dominicana, Jamaica, y los países organizados en la Organización de Estados del Caribe Oriental.

El PCLR tiene como objetivo central promover la sistematización, diseminación y uso de evidencias de investigación y recursos para aumentar el impacto, la calidad y la sostenibilidad de las políticas y prácticas de lectoescritura inicial (LEI) en los países en los que trabaja.
Para ello organizamos un conjunto de procesos:

- Identificamos programas, prácticas y políticas exitosas en la región.

- Realizamos una revisión sistemática de la evidencia existente en el campo de la LEI.

- Recopilamos documentos y recursos pedagógicos que se han usado en la región para enseñar la LEI en los últimos diez años.

- Identificamos a los actores claves en los países priorizados por el programa y describimos sus intervenciones, su interés y su influencia en este campo.

- Diseminamos materiales y evidencias para mejorar la enseñanza en base a los requerimientos de los países.

- Articulamos en cada país un plan de creación de capacidades en función de su contexto específico y sus necesidades particulares.

- Promovemos la articulación de redes nacionales y regionales para promover la investigación, la generación de evidencias y el intercambio.

- Participamos, con nuestros socios, en diversos foros nacionales y regionales que discuten acerca de LEl, entre ellos el Congreso Latinoamericano de Lectura y Escritura (CONLES) organizado por el Comité Latinoamericano para el Desarrollo de la Lectura y la Escritura, miembro de la International Literacy Association.

En este artículo presentamos los principales aprendizajes del Programa y compartimos los desafíos actuales.

\section{LÍMITES DEL PROCESO}

Los resultados a presentar no son hallazgos de una investigación, sino resultados de procesos de trabajo desarrollados con distintas metodologías y en distintos universos, por tanto, las conclusiones deben ser leídas teniendo en cuenta los límites que este proceso establece. 


\section{METODOLOGÍAS}

El análisis sistemático de la evidencia (Early Grade Reading in Latin America and the Caribbean:ASystematic Review.) fue realizado con una metodología clara y consistente, mediante una búsqueda exhaustiva de evidencia regional; sin embargo, el Análisis de Actores Clave se basó en las percepciones de los entrevistados. Por su parte, el análisis de la participación en los eventos, se sustenta en las opiniones de nuestro equipo y los socios locales.

\section{UNIVERSO}

El universo de trabajo ha sido diferente. El Systematic Review se hizo en toda la región, es decir, en más de cuarenta países con múltiples idiomas, poblaciones diversas y sistemas educativos con diferente organización y definiciones; con una sobre representación de los países de ingresos medios y altos. Por su parte, el Análisis de Actores Clave, únicamente se hizo en los países con los que directamente trabajamos en el Programa, que están ubicados entre los de más bajos ingresos. El Análisis de las Ponencias e Investigaciones presentadas en CONLES y otros foros, se realizó con el universo de los participantes en este foro, una minoría en el continente, solamente hispanoparlantes y algunos brasileños, con la ausencia de países como Honduras y El Salvador.

\section{PRINCIPALES APRENDIZAJES}

A continuación, presentamos algunos de los aprendizajes de este proceso.

\section{Se han identificado progresos}

A pesar de los problemas, hemos encontrado que en algunos países hay importantes progresos en los últimos años, entre los principales están:

- Trabajo pionero en aprendizaje en lengua materna.

- Instalación de sistemas permanentes de evaluación de aprendizajes.

- Diseño y utilización de materiales basados en las más recientes evidencias disponibles.
- Impulso de programas de desarrollo profesional para docentes en el que se debaten evidencias internacionales y se comparten enfoques novedosos.

\section{CONCIENCIA DE LAS CARENCIAS}

Existe conciencia de las carencias, incluso de los ministerios de educación, lo que ha contribuido a la demanda de apoyo para la creación de capacidades locales.

\section{RED DE ACTORES IMPORTANTE}

Existe una importante red de actores que trabajan en LEI en los países de la región, provenientes del sector público, organismos internacionales, organizaciones no gubernamentales internacionales, la academia, instituciones formadoras de docentes y organizaciones no gubernamentales nacionales. En cada país, la influencia y el interés de los actores varía, sin embargo, se observa un interés creciente de gobiernos e instituciones por el tema.

Los actores principales conocen bastante bien sus limitaciones y fortalezas y valoran de manera especial la colaboración y el intercambio entre ellos:

- Consideran el trabajo y la articulación en redes como la principal estrategia para potenciar sus intervenciones.

- Piensan que el trabajo en red es una buena estrategia para ampliar los espacios de diálogo entre políticos e investigadores, espacios que actualmente son muy limitados.

- Les permite más fácilmente identificar cuellos de botellas y temas relevantes para una agenda de investigación.

- Maximiza las posibilidades de movilizar recursos hacia las necesidades de investigación, creación de capacidades, intervenciones y en definitiva las posibilidades de aumentar sus esferas de influencia en la política educativa nacional.

AUSENCIA DE PRÁCTICA DE PRODUCCIÓN DE EVIDENCIAS LOCALES

En general, en la región se encontraron pocos estudios que afirmen la existencia de relaciones causales creíbles entre programas y estrategias de 
desarrollo dentro y fuera del aula en los resultados de LEI (LAC Reads Capacity Program (LRCP), 2016a). Los actores claves de los países expresan que, en su opinión, no se ha dado prioridad a identificar lo que mejor funciona para aprender y enseñar a leer con éxito en los primeros grados (identificar las "evidencias locales") y que podría ser diferente de lo que funciona en otras regiones del mundo (LRCP, 2016b). De hecho, ellos no hacen referencia al uso de evidencias basada en investigación para informar de su trabajo y tomar decisiones.

En diferentes foros a los que hemos asistido, entre ellos CONLES, observamos que muchas de las presentaciones tendían a seguir un formato informal que parecía estar diseñado para compartir las experiencias de los presentadores, en lugar de seguir una metodología de investigación rigurosa que pudiera demostrar el impacto. Por ejemplo, muchas presentaciones parecían estar limitadas a las experiencias de los docentes en el aula y debido a sus marcos conceptuales y metodologías insuficientemente definidas, su aplicabilidad a otros contextos se consideraba bastante limitada (LRCP, 2017).

Esta falta de investigación que genera evidencia, es aún mayor en Centroamérica y el Caribe. En CONLES la mayoría de los presentadores provenían principalmente de varios países de América del Sur, así como de México. Por ejemplo, un análisis del programa de dicho Congreso mostró que todas las presentaciones dentro del ámbito de desarrollo profesional docente fueron extraídas de México ( $L R C P$, 2017). Esta realidad hace eco de los resultados de la revisión sistemática hecha por el PCLR, que encontró que solo el $5 \%$ de la investigación proviene de Centroamérica y solo el $12 \%$ del Caribe (LRCP, 2016a).

Adicional a la falta de investigación, hay un vacío en los temas de investigación y en el rigor de la misma. La mayoría de los estudios de intervención cuantitativa tienen un riesgo medio o alto de sesgo de selección o un riesgo medio o alto de sesgo de rendimiento, lo que significa que la evidencia se deriva de estudios metodológicamente poco rigurosos (LRCP, 2016a).

Además, encontramos pruebas contundentes del sesgo de publicación en los estudios que se centran en los efectos de las prácticas de los docentes y la participación de los padres en los resultados de LEl es decir, es probable que haya una gran cantidad de estudios adicionales que no se hayan publicado sobre temas similares porque no encontraron efectos estadísticamente significativos (LRCP, 2016a).

No encontramos publicaciones sobre intervenciones no efectivas (LRCP, 2016a). Es importante señalarlo puesto que cuando solamente se publican los resultados positivos y estadísticamente significativos, se impide a los responsables de formular políticas, y tomar decisiones basadas en la evidencia.

Encontramos sólo unos pocos artículos sobre cada tema, y el tamaño de las muestras es pequeño, por lo que los resultados necesitan estudios adicionales para ser validados (LRCP, 2016a). Por lo tanto, nuestra síntesis sugiere que la evidencia actual que muestra el impacto de las intervenciones en LEl, es bastante pequeña. Esto no es inusual puesto que sabemos que la tendencia a realizar evaluaciones de impacto de las intervenciones y programas es reciente (Cameron, Mishra \& Brown, 2015).

También sabemos que muchas investigaciones y artículos no fueron publicados por lo que es difícil acceder a ellos y constituyen lo que podemos llamar "literatura gris". Estos no formaron parte de esta revisión.

Se constató que existe en la región, un desafío relacionado con la disponibilidad y el uso de materiales para la enseñanza de la lectoescritura. Hay consenso entre los actores claves (LRCP, 2016b; LRCP, 2017) sobre la necesidad de abordar la falta de acceso a materiales de alta calidad, contextualmente relevantes y asequibles en toda la región. Por ejemplo, en CONLES los stands de las exhibiciones de la conferencia eran editoriales privadas que ofrecían muy pocas opciones en los materiales que exhibían (LRCP, 2017). La mayoría solo vendían libros para niños y había una escasa disponibilidad de material pedagógico o de desarrollo profesional a la venta. Además, dado que la audiencia estaría muy interesada en los libros que podrían distribuirse a gran escala entre las poblaciones desfavorecidas, los materiales parecían tener un costo prohibitivo $y$, por lo tanto, en gran medida irrelevantes para la audiencia prevista (LRCP, 2017). 
Por otra parte, muchas de las universidades e instituciones formadoras de docentes, en la mayoría de los países de Centroamérica, muestran poco involucramiento y compromiso en la investigación y experimentación de nuevos modelos en LEl, a pesar del consenso regional en torno al papel relevante de los docentes en el avance de los resultados del aprendizaje y del papel crucial que juega el buen aprendizaje de la lectoescritura en los primeros grados para lograr una educación de calidad.

\section{POCA CLARIDAD ALREDEDOR DE LOS MARCOS CONCEPTUALES}

Parece no existir suficiente claridad alrededor de los marcos conceptuales en LEl que subyacen al diseño de intervenciones, diseño de materiales y programas de formación y especialmente no se encuentra un esfuerzo conceptual por construir conocimiento a partir de los resultados de buenas prácticas y de programas exitosos. Tampoco hay esfuerzos por generar espacios de debate que ayude a los educadores y tomadores de decisión de la región a avanzar en este campo.

Todo ello, pese a que existen, al menos, dos enfoques diferentes en el continente, uno el de la llamada corriente "constructivista latinoamericana" que plantea la necesidad de un aprendizaje de la lectura contextualizada, la promoción del aprendizaje por descubrimiento y la necesidad de comprender el pensamiento del niño para poder enseñar. No se trata exactamente de lo que planteaba hace algún tiempo el llamado Método Global, puesto que ahora se propone en algunas etapas la enseñanza explícita de palabras. Por otro lado, se nota una influencia del enfoque originado en los estudios del National Reading Panel (2000) de los Estados Unidos basado en el desarrollo de cinco o seis habilidades (NICHD, 2000).

Tampoco parece haber un debate entre las muy diferentes corrientes de enseñanza de la lectoescritura basadas en la neurociencia. En CONLES, por ejemplo, se presentaron varias teorías neuro-científicas, basadas en investigaciones rigurosas y generalmente bien recibidas por el público, pero presentaban enfoques y planteamientos a veces contradictorios y esto no fue aprovechado para generar un debate académico de altura y calidad que tanta falta hace en la región (LRCP, 2017).
Adicionalmente, mucha conceptualización teórica tiene problemas para aterrizarse en la práctica. Por ejemplo, hemos observado que en muchos foros, los presentadores afirman que la comprensión lectora es un factor importante asociado con el éxito académico, pero no proporcionan evidencias de estrategias específicas o componentes del plan de estudios que los asistentes puedan utilizar para mejorar su propia práctica (LRCP, 2017).

\section{RETOS DE LA LECTOESCRITURA}

Claramente hay un problema continental con la Comprensión Lectora sin embargo el tema no se ha estudiadoy experimentado suficientemente por lo que no hay claridad sobre cómo mejorar estas habilidades crítica para el aprendizaje de la lectoescritura.

Hay conciencia del problema, sin embargo, falta atención y debate sobre los pasos que podrían tomarse para mejorar los niveles de comprensión de lectura en la región. En los foros internacionales en los que hemos participado, la delegación de PCLR notó que muy pocas de las presentaciones abordaron la necesidad de enseñar explícitamente estrategias de lectura a los niños, ni se planteó la necesidad de desarrollar capacidades entre los maestros en esta área (LRCP, 2017).

\section{AUSENCIA DE DEBATE SOBRE LAS INTERVENCIONES EDUCATIVAS MULTILINGÜES}

En la mayoría de los países parece haber una ausencia de debate sobre las intervenciones educativas multilingües y las políticas de instrucción en la lengua materna.

Los resultados de la recolecta de recursos en LEI en los países de interés del programa (casi todo Centroamérica y República Dominicana, sin incluir a Guatemala) muestran que únicamente el $0.33 \%$ de los recursos recolectados se encuentra en lenguas locales (LRCP, 2018). Este dato refleja un aspecto problemático que encontramos en la enseñanza de la lectura y la escritura en la educación primaria en Nicaragua, Honduras, Jamaica y República Dominicana: la falta de políticas y esfuerzos para enseñar a leer y escribir en la lengua materna en los primeros grados. Esta podría ser una de las principales causas de los problemas 
de aprendizaje identificados por las evaluaciones nacionales e internacionales, especialmente entre los grupos más vulnerables, como los niños migrantes haitianos en la República Dominicana o los pueblos originarios en Nicaragua y Honduras.

En CONLES, no hubo un seguimiento específico de las políticas lingüísticas o multilingües. Los participantes de PCLR identificaron únicamente dos presentaciones que discutieron varios desafíos relacionados con la educación multilingüe en la región (LRCP, 2017). Sin embargo, solo hubo una presentación, de un total de 161 , que incluyó cualquier forma de multilingüismo o bilingüismo en su título (LRCP, 2017). Dada la relevancia e importancia de este tema en la región, los participantes de PCLR esperaban que el tema en general atrajera más atención en la conferencia a través de discusiones y debates y específicamente que una mayor proporción de las presentaciones se habría centrado directamente en varios enfoques pedagógicosydepolítica que seestán implementando.

\section{SIMILITUDES CON OTRAS REGIONES DEL MUNDO}

Los hallazgos de nuestra Revisión Sistemática de la literatura parecen ser muy comparables con los de otras, en todo el mundo:

- El aprendizaje social y la consideración del contexto son claves para mejorar los resultados de la lectura. Los artículos en nuestras síntesis discuten específicamente el contexto y la experiencia de trabajo infantil, pobreza y nutrición, la importancia de la familia y la consideración de los estudiantes en el proceso de aprendizaje (LRCP, 2016a).

- Los enfoques de aprendizaje colaborativo brindan una oportunidad para que los estudiantes se ayuden unos a otros a construir significado y conocimiento.

- Los programas de capacitación de los maestros pueden tener un efecto positivo en los resultados de lectura inicial en países de ingreso alto y mediano alto, cuando se ejecutan bien y se complementan con acompañamiento en el aula.
- Los programas de nutrición pueden tener efectos positivos en los resultados de lectura en los primeros grados en contextos en que las tasas de retraso del crecimiento y desnutrición son altas entre los niños y las niñas.

- Los programas que se centran en proporcionar tecnología de la información y las comunicaciones, sin hacer énfasis en la pedagogía, no logran mejorar los resultados de lectura en los primeros grados.

- La conciencia fonémica, la fonética, la fluidez y la comprensión se asocian con la capacidad de lectura.

- La pobreza, la malnutrición y el trabajo infantil tienen una correlación negativa con los resultados de lectura en los primeros grados.

- La calidad de la educación preescolar se asocia de manera positiva con los resultados de lectura en los primeros grados.

Pensamos que el tamaño reducido de la muestra no permitió profundizar en aspectos propios de la región, que aún no se han sido explorados. Esto nos plantea un desafío en el desarrollo de procesos investigativos y publicaciones de calidad que nos permitan afinar la búsqueda de factores adicionales.

\section{NECESIDADES DE CREACIÓN DE CAPACIDADES}

Los diversos procesos impulsados por PCLR coinciden en identificar las siguientes necesidades de creación de capacidades:

- Formación sobre metodologías o estrategias de aula que promueven las seis habilidades esenciales para aprender a leer y escribir.

- Estrategias explícitas para fortalecer las habilidades de comprensión lectora.

- Modelado de prácticas efectivas y apoyo en la planeación didáctica para guiar la enseñanza y la sistematización y divulgación de prácticas efectivas para desarrollar los procesos de aprendizaje - enseñanza de la lectoescritura en los primeros grados.

- Desarrollo de indicadores y herramientas para evaluar los resultados, para analizar el currículo, los materiales educativos y las prácticas de aula. 
- Enseñanza en la lengua materna, educación multicultural y otras intervenciones efectivas para abordar las brechas de alfabetización inicial entre los grupos indígenas y otros grupos minoritarios.

- Modelos de formación inicial y en servicio de docentes en LEI.

- Formación para mejorar las capacidades de investigación conducente a producir evidencias locales.

- Apoyo para incrementar las capacidades de publicar los resultados de investigaciones y evaluaciones en revistas indexadas.

- Trabajo con familias y comunidades en apoyo a la alfabetización inicial.

- Promoción de las habilidades de lenguaje oral y de prelectura y pre-escritura en la educación preescolar.

- Estrategias y enfoques para estudiantes dotados y talentosos, así como para estudiantes con discapacidades diversas.

\section{PARA UNA AGENDA DE INVESTIGACIONES}

En estos años de trabajo en la región encontramos las siguientes preguntas que pueden orientar la articulación de una agenda regional de investigación:

1. ¿Existen vínculos entre el desarrollo de las destrezas de pre-escritura y escritura y los resultados de la lectoescritura inicial? Si es así, ¿qué tipos de destrezas pronostican mejor los resultados de la lectoescritura inicial?

2. ¿Cómo podemos apoyar un desarrollo más eficaz de las destrezas de lectoescritura en niños de orígenes lingüísticos diversos (incluidos el desarrollo de la lectoescritura en lengua materna y el desarrollo de resultados de lectoescritura bilingües y multilingües)?

3. ¿Cuál es el umbral que define cuándo los niños están listos para una transición entre la primera lengua y la segunda lengua? ¿Qué nivel de habilidades necesitan en su primera lengua y qué nivel de vocabulario oral necesitan en su segunda lengua para una transición efectiva?
4. ¿De qué manera se puede reconocer eficazmente a los estudiantes que tienen diversas discapacidades y cuáles son las estrategias de lectoescritura inicial que apoyan el mejoramiento de sus resultados?

5. ¿Cuáles son los efectos causales de programas específicos de desarrollo en los resultados de lectoescritura inicial?

6. ¿Cuáles son los efectos de la educación preescolar y de la primera infancia en los resultados de lectoescritura inicial?

7. ¿Cuáles son los factores del contexto escolar y social que tienen mayor impacto en la mejora de la comprensión lectora, un problema neurálgico en la región?

8. ¿Cuáles son los modelos de formación inicial, en servicio y coaching más efectivos para mejorar las prácticas de aula de los docentes y los resultados de aprendizaje de los estudiantes?

9. ¿Cuáles métodos y enfoques de instrucción en LEI son más efectivos en el aprendizaje temprano de los estudiantes?

Los actores y socios del programa en la región, han identificado otros temas, siendo los principales:

- Los factores que pueden predecir de manera temprana (predictores) el éxito en comprensión lectora y a los que, por lo tanto, hay que dar especial atención en preescolar y en los primeros grados.

- Evaluación del Estado de la Región en Lectoescritura Inicial en América Central.

- Resultados de la alfabetización entre niños y niñas que no asisten a la escuela

- Estrategias efectivas para desarrollar habilidades de pensamiento crítico entre estudiantes de los primeros grados.

- Modelos de remediación efectivos y eficientes para estudiantes con dificultades.

- El impacto de los programas que se centran en la promoción de las tecnologías de la información y comunicación (TIC) en los resultados del aprendizaje de la lectoescritura temprana. 
- El desarrollo de investigaciones interdisciplinarias que incluyan métodos mixtos en el campo de la lectoescritura temprana y más de una habilidad importante para el aprendizaje de la lectoescritura en muestras de gran tamaño. Estos estudios también deberían ser capaces de responder a preguntas hipotéticas para determinar, de manera creíble, los efectos de los programas que prueban o evalúan los resultados en lectoescritura temprana. La investigación interdisciplinaria permitirá a los investigadores examinar los mecanismos que subyacen a los cambios que observen, mediante la incorporación de estimaciones de lectura y de impacto cualitativo en más de una habilidad de lectoescritura.

Las preguntas y temas resumidos anteriormente forman parte de los desafíos que enfrenta el campo de la LEl en la región y que necesita atención e inversión de recursos por parte de los diferentes actores clave de cada país y especialmente de parte de los ministerios de educación, la academia y las agencias de desarrollo internacional.

\section{RECOMENDACIONES ORIENTADAS POR LA EVIDENCIA}

El consenso general sobre la falta de investigación rigurosa ilustra tanto el mayor reconocimiento de esta brecha, como la mayor demanda de investigación de alta calidad. Debido a lo anterior, nuestros socios identifican el impulso de investigaciones y la divulgación de resultados de lo que se produce en el mundo y en la región, como un paso importante para empujar en la dirección de producir evidencias y basarse en las mismas para diseñar políticas e intervenciones. Esta es una oportunidad única para que los diferentes actores nacionales, regionales e internacionales, como el PCLR, avancen en su trabajo en la región.

Para tener mejores resultados de la investigación, se requiere:

1. Concebir investigaciones interdisciplinarias con métodos mixtos en las que se incluya más de un modelo de datos para lectoescritura y muestras más grandes.
2. Incluir la recolecta de datos de lectoescritura inicial en los datos administrativos nacionales.

3. Efectuar investigaciones experimentales o cuasiexperimentales con métodos mixtos sobre efectos de la educación preescolar y de la primera infancia en los resultados de lectoescritura en los primeros grados.

4. Documentar y registrar en un lugar central y de acceso público, las investigaciones en curso.

5. Hacer un seguimiento de la investigación en progreso y apoyar para que su información sea accesible.

Para hacer realidad estas investigaciones, se requiere de un esfuerzo financiero de parte de los gobiernos, las universidades, las organizaciones locales y las agencias internacionales.

\section{POSIBLES OPORTUNIDADES PARA EXPANDIR LAS ACTIVIDADES DEL PCLR EN LA REGIÓN}

Dadas las tendencias regionales, las áreas de fortaleza, así como las debilidades identificadas, hay varias áreas potenciales donde el PCLR podría expandirse y desarrollar su trabajo en la región:

1. Desarrollar la capacidad para llevar a cabo investigaciones rigurosas y comunicar resultados de manera efectiva a audiencias diversas. Dada la necesidad de producir evidencias locales surgidas de investigación rigurosa, se requiere construir capacidades y demandas para las evaluaciones que midan el impacto de intervenciones específicas en los resultados de LEl. Al mismo tiempo, los datos deberán comunicarse de manera efectiva a tres públicos distintos: a (1) otros investigadores académicos, (2) tomadores de decisiones y (3) el público en general. Por lo tanto, una oportunidad potencial para el Programa de Capacidad LAC Reads sería también desarrollar la capacidad regional para comunicar y diseminar de manera efectiva los resultados de la investigación, para contribuir a que los datos se traduzcan en políticas significativas y decisiones programáticas. 
2. Crear espacios para que los expertos regionales de LEl compartan regularmente las mejores prácticas y las lecciones aprendidas y debatan ideas diferentes sobre el tema de tal manera que se construya conocimiento regional. EI CONLES convoca a expertos cada dos años, pero se sugirió que el PCLR podría asumir un papel más activo en la creación y promoción de oportunidades frecuentes de discusión entre los diversos actores dentro de la región. Por ejemplo, una conferencia o reunión internacional con actores claves de cada país socio para presentar evidencia y discutir las mejores prácticas en LEl. Tal esfuerzo promovería la sostenibilidad al generar una mayor sinergia entre los actores de la región.

3. Construir conexiones regionales más allá de los países PCLR. El Programa ha recibido numerosas demandas de otros países de la región como Costa Rica y Panamá, quienes expresaron su interés en fomentar las relaciones con las organizaciones asociadas de PCLR. Dadas las similitudes de muchos de los desafíos en LEl en la región, varios participantes sugirieron que un enfoque de investigación regional podría ser una estrategia efectiva para desarrollar capacidades y lograr un mayor impacto. Dado el corto tiempo que queda en el proyecto, este es un desafío que podría asumir la Red de Lectoescritura de Centroamérica y República Dominicana (RedLEl) que se ha organizado desde Guatemala para trabajar con una visión regional, para promover y mantener las conexiones regionales.

4. Mayor difusión de las investigaciones y recursos de PCLR como una manera de diseminar evidencias y literatura especializada en el tema que permita avanzar el pensamiento regional en LEI.

5. Formación para ayudar a los maestros a comprender cómo integrar varios enfoques para la enseñanza basada en evidencia. Dada la gran cantidad de enfoques y métodos existentes, los docentes y otros responsables de la toma de decisiones pueden sentirse abrumados con la forma de utilizar la investigación para mejorar sus prácticas diarias. Por lo tanto, un área importante de consideración para el proyecto puede ser desarrollar modelos de capacitación a docentes que les permitan entender la evidencia y saber cómo integrar los diversos enfoques de manera coherente en sus clases.

\section{ACTIVIDADES DE CREACIÓN DE CAPACIDAD DE PCLR}

Sobre la base de las necesidades identificadas en el Análisis de Actores Claves, cada socio del PCLR desarrolló una estrategia nacional de creación de capacidad que incluyen un conjunto de talleres y foros de discusión a desarrollarse en los últimos dos años del proyecto. Algunos de estos eventos fueron:

1. En la República Dominicana, en junio de 2018 se llevó a cabo un taller sobre el uso de la Jornada Extendida para fortalecer las habilidades de LEI de los niños.

2. En Honduras, Ferema organizó dos eventos. EI primer evento se centró en la sistematización de varias experiencias exitosas de LEl en el país y su segundo evento, el Encuentro Internacional III, convocó e involucro a varios actores locales sobre el tema de LEI.

3. En Guatemala, ASIES organizó un seminario internacional titulado "Importancia de asumir el enfoque de uso de evidencias para diseñar intervenciones en LEI". Durante este evento, diferentes organizaciones nacionales e internacionales presentaron los resultados de sus evaluaciones.

4. En Nicaragua, CIASES diseñó y comenzó a recopilar datos cualitativos y cuantitativos para estudiar la implementación del método FAS (Fonético, Analítico, Sintético), que es el método único prescrito por el Ministerio de Educación para enseñar a leer y escribir en los primeros grados.

5. En Jamaica, EduconnectJA implementó dos talleres que reunieron a varios actores clave para desarrollar materiales de lectura que son relevantes para el contexto en Jamaica para estudiantes en los primeros años. Además, en septiembre de 2018, se llevó a cabo la Conferencia de Lectoescritura Inicial, titulada "Get Reading Right from the Start!". Varios cientos de personas asistieron al evento.

Además de las actividades nacionales de creación de capacidad, el LRCP identificó áreas comunes entre todos los socios, con las cuales el proyecto diseñaría e implementaría eventos. Las dos áreas que 
se identificaron inicialmente fueron: (1) estrategias y enfoques para apoyar el desarrollo de habilidades de prelectura y preescritura entre niños de cero a seis años y; (2) un curso de desarrollo profesional centrado en los fundamentos de lo que dice la evidencia sobre cómo los niños aprenden a leer. Entre julio y octubre de 2018, el LRCP implementó una conferencia y un taller que se centró en las habilidades de prelectura y preescritura en Honduras, Guatemala y la República Dominicana. Además, el proyecto, en colaboración con Juárez y Asociados, ha diseñado un curso sobre lectoescritura inicial que comenzará en octubre de 2018. Las personas de cada país del proyecto participarán en el curso de 10 semanas de duración.

\section{CONCLUSIÓN Y DISCUSIÓN}

La región vive un momento único en su historia. La demografía nos ofrece oportunidades que nunca antes habíamos vislumbrado, sin embargo, aprovecharlas depende de las políticas educativas y oportunidades laborales que impulsen los países. Lograr una educación de calidad accesible para todos, es la clave que lo hará posible puesto que como lo señala la estrategia de la educación de USAID: "A menos que haya progresos significativos en la calidad, el enorme poder de la educación para mejorar las vidas de las personas y las empresas se continuará desperdiciando" (USAID, 2011, p.7). La base para lograr la calidad de la educación es la lectoescritura. Lograr que los niños y las niñas aprendan a leer y escribir bien en los primeros grados es una condición de éxito para lograr la calidad, y por tanto debe ser la prioridad de toda política educativa y social, y el ámbito al que se dediquen más esfuerzos de parte de organizaciones de la sociedad civil, empresas, organismos internacionales, universidades y familias. De la manera en que enfrentemos los desafíos que nos plantea el buen aprendizaje de la lectura y la escritura, depende nuestro futuro como región.

\section{REFERENCIAS}

Cameron, D. B., Mishra, A., \& Brown, A. N. (2015). The growth of impact evaluation for international development: how much have we learned? Journal of Development Effectiveness, 8(1), 1-21.

CIASES. (2016). Nicaragua: Perfil del país y análisis de actores clave en lectoescritura inicial. Managua: USAID - LRCP.

EduConnectJA. (2016). Jamaica: country profile and analysis of stakeholders in early grade literacy. Kingston: USAID - LRCP.

FEREMA. (2016). Honduras: perfil del país y análisis de actores clave en lectoescritura inicial. Tegucigalpa: USAID - LRCP.

LAC Reads Capacity Program. (2016a). Early grade reading in latin america and the caribbean: a systematic review. Washington D.C.: USAID - LRCP.

LAC Reads Capacity Program. (2016b). Early grade literacy in latin america and the caribbean: stakeholder mapping and analysis. Washington D.C.: USAID - LRCP.

LAC Reads Capacity Program. (2017). Tendencias latinoamericanas en lectoescritura inicial identificadas en el congreso latinoamericano para el desarrollo de la lectura y escritura. Washington D.C.: USAID - LRCP.

LAC Reads Capacity Program. (2018). Resources database [database]. Retrieved from https:// lacreads.org/database/resources. 
NICHD. (2000). National Reading Panel (US), National Institute of Child Health, \& Human Development (US). (2000). Report of the national reading panel: Teaching children to read: an evidence-based assessment of the scientific research literature on reading and its implications for reading instruction: Reports of the subgroups. National Institute of Child Health and Human Development, National Institutes of Health. Retrieved November, 2017 from: from https://www.nichd.nih.gov/publications/pubs/ nrp/documents/report.pdf

USAID. (2011). Estrategia de educación USAID. 2011 -2015. Oportunidad a través del aprendizaje. Washington D.C.: USAID. 